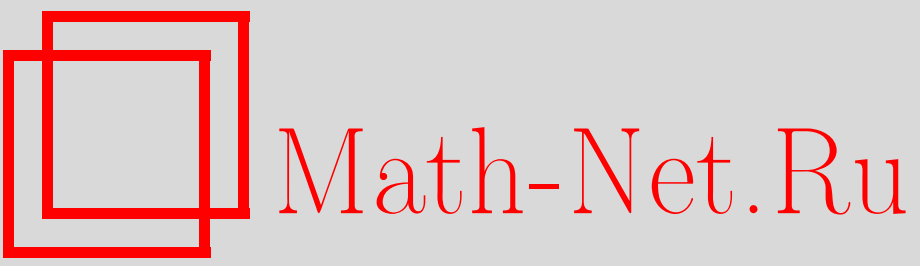

А. И. Аптекарев, А. Дро, В. А. Калягин, Об асимптотике точных констант в неравенствах Маркова-Бернштейна в интегральных метриках с классическим весом, УМH, 2000, том 55, выпуск 1, 173-174

DOI: https://doi.org/10.4213/rm251

Использование Общероссийского математического портала Math-Net.Ru подразумевает, что вы прочитали и согласны с пользовательским соглашением

http://www.mathnet.ru/rus/agreement

Параметры загрузки:

IP : 3.85 .183 .62

26 апреля 2023 г., $17: 44: 48$ 


\title{
ОБ АСИМПТОТИКЕ ТОЧНЫХ КОНСТАНТ В НЕРАВЕНСТВАХ МАРКОВА-БЕРНШТЕЙНА В ИНТЕГРАЛЬНЫХ МЕТРИКАХ С КЛАССИЧЕСКИМ ВЕСОМ
}

\author{
А.И. Аптекарев, А. Дро, В. А. КаляГИН
}

Пусть $X, Y$ банаховы пространства непрерывных функций действительного переменного, содержащие многочлены. Неравенство Маркова-Бернштейна имеет вид

$$
\left\|P^{\prime}\right\|_{X} \leqslant M_{n}\|P\|_{Y}, \quad \operatorname{deg} P \leqslant n .
$$

Константа $M_{n}$ называется наилучшей, если неравенство нельзя улучшить для всех многочленов степени не выше $n$. Для наилучшей константы имеем очевидно $M_{n}=M_{n}(X, Y)=$ $\sup \left\{\left\|P^{\prime}\right\|_{X} /\|P\|_{Y}, \operatorname{deg} P \leqslant n\right\}$. Классическими примерами являются неравенство Маркова для алгебраических многочленов в пространствах $X=Y=L^{\infty}[-1,1]$ с наилучшей константой $M_{n}=n^{2}$ и неравенство Бернштейна для тригонометрических многочленов в пространствах $X=Y=L^{\infty}[-\pi, \pi]$ с наилучшей константой $M_{n}=n$ (см. [1]). Неравенства Маркова-Бернштейна известны для большого набора пространств $X, Y$. Проблема определения наилучшей константы является более сложной и результаты в этом направлении не столь многочисленны (см. энциклопедический обзор в [2]). В настоящей работе рассматриваются неравенства типа (1) для алгебраических многочленов в весовых функциональных пространствах $X=Y=L^{2}([a, b], w(x))$ с классическим весом $w(x)$. Известны результаты для весовых функций Эрмита, Лагерра и Лежандра [2]:

1. $w(x)=\exp \left(-x^{2}\right), x \in(-\infty, \infty), M_{n}=\sqrt{2 n}$ (E. Schmidt, 1944);

2. $w(x)=\exp (-x), x \in[0, \infty), M_{n}=\frac{1}{2 \sin (\pi /(4 n+2))}(\mathrm{P}$. Turan, 1960);

3. $w(x)=1, x \in[-1,1]$, наилучшая константа неизвестна, но известна ее асимптотика $M_{n} \sim(2 n+3)^{2} / 4 \pi$ (E. Schmidt, 1944).

Основным резултатом настоящей работы являются асимптотики наилучших констант в неравенстве Маркова-Бернштейна для случая обобщенного веса Лагерра $w(x)=x^{\alpha} \exp (-x)$, $x \in[0, \infty), \alpha>-1$ и весов Гегенбауэра $w(x)=\left(1-x^{2}\right)^{\gamma}, x \in[-1,1], \gamma>-1$.

Tеорема 1. Пусть $X=Y=L^{2}([a, b], w(x))$, moгдa

1. Для $w(x)=x^{\alpha} \exp (-x),[a, b]=[0, \infty)$ наилучшая константа в неравенстве Маркова-Бернштейна (1) имеет асимптотику

$$
M_{n}=\frac{n}{x_{1}}[1+O(1)]
$$

где $x_{1}$ - ближайший $\kappa$ началу координат ноль функции Бесселя $J_{\beta}(x)$, $\beta=(\alpha-1) / 2$.

2. Для $w(x)=\left(1-x^{2}\right)^{\gamma},[a, b]=[-1,1]$ наилучшая константа в неравенстве Маркова-Бернштейна (1) имеет асимптотику

$$
M_{n}=\frac{n^{2}}{2 x_{1}}[1+O(1)]
$$

где $x_{1}$ - ближайший $\kappa$ началу координат ноль функции Бесселя $J_{\beta}(x)$, $\beta=(\gamma-1) / 2$.

ДокАЗАТЕльство. В литературе известны два подхода к определению наилучшей константы в пространствах вида $L^{2}([a, b], w(x))$. Первый подход, развитый Миловановичем (см. [2]), состоит в приведении к диагональному виду матришы оператора дифференцирования в базисе ортогональных с весом $w(x)$ многочленов. Наилучшая константа тогда связана с экстремальными собственными значениями этой матрицы. Другой подход предложен в работе [3], где рассматривается задача об определении наименьшего (отрицательного) значения константы $\lambda$ при котором квадратичная форма

$$
\langle p, q\rangle:=\int p(x) q(x) w(x) d x+\lambda \int p^{\prime}(x) q^{\prime}(x) w(x) d x
$$


остается положительно определенной в линейном пространстве многочленов степени $n$. Для случая пространств с классическими весами оба подхода приводят к исследованию экстремальных нулей последовательности многочленов определенной симметричным рекуррентным соотношением четного порядка (см. подробности в [2], [3]). В случае обобщенного веса Лагерра $w(x)=x^{\alpha} \exp (-x)$, например, соответствующая последовательность многочленов определяется равенствами:

$$
Q_{n}(x)=\left(x-2-\frac{\alpha}{n}\right) Q_{n-1}(x)-\left(1+\frac{\alpha}{n-1}\right) Q_{n-2}(x), \quad n \geqslant 2,
$$

с начальными условиями $Q_{0}(x)=1, Q_{1}(x)=x-\alpha-1$. В этом случае для наилучшей константы имеет место равенство $M_{n}=1 / \sqrt{\mu_{1, n}}$, где $\mu_{1, n}$ - ближайший к началу координат ноль многочлена $Q_{n}(x)$. Для Якоби рекуррентное соотношение имеет порядок 4 (многочлен степени $n$ выражается через 4 предыдущих многочлена). В случае веса Гегенбауера каждый многочлен последовательности является произведением двух многочленов, для определения которых известны рекуррентные соотношения 2-го порядка. Явные формулы смотри в работе [3]. Задача об асимптотике наилучшей константы сводится, таким образом, к задаче об асимптотике экстремального нуля последовательности многочленов, определенных некоторым рекуррентным соотношением 2 -го порядка. Общий результат в этом направлении получен в работе [4]. Для исследования асимптотики мы следуем, в основном, идее этой работы. Рассмотрим случай обобщенного веса Лагерра. Нас интересует поведение многочленов $Q_{n}(x)$, определенных равенствами $(2)$ в окрестности точки $x=0$. Выберем иную нормировку и перейдем к последовательности многочленов $q_{n}(x)=Q_{n}(x) / Q_{n}(0), n \geqslant 0$. Для новой последовательности выполнено $q_{n}(0)=1$ и многочлены связаны рекуррентным соотношением

$$
x q_{n}(x)=a_{n} q_{n+1}(x)+b_{n} q_{n}(x)+c_{n} q_{n-1}(x),
$$

где $a_{n}=Q_{n+1}(0) / Q_{n}(0), b_{n}=2+\alpha /(n+1), c_{n}=Q_{n-1}(0) / Q_{n}(0)(1+\alpha / n)$. Прямые вычисления дают $Q_{n+1}(0) / Q_{n}(0)=-(1+\alpha /(n+1))$. Используя этот факт преобразуем разностное уравнение (3) к виду

$$
\frac{q_{n+1}^{h}-2 q_{n}^{h}+q_{n-1}^{h}}{h^{2}}+\frac{2 \alpha}{z} \frac{q_{n+1}^{h}-q_{n-1}^{h}}{2 h}+q_{n}^{h}=O\left(\frac{1}{n}\right),
$$

которое получается из (3) при $x=h^{2}, n h=z, q_{n}^{h}=q_{n}\left(h^{2}\right)$. Аккуратный переход к пределу при $n \rightarrow \infty(h \rightarrow 0)$ переводит разностное уравнение $(4)$ в дифференциальное $y^{\prime \prime}(z)+$ $(2 \alpha / z) y^{\prime}(z)+y(z)=0$, решением которого (с начальным условием $\left.y(0)=1\right)$ является функция $j_{\beta}(z)=2^{\beta} \Gamma(\beta+1) z^{-\beta} J_{\beta}(z)$, где $J_{\beta}$ стандартная функция Бесселя, $\beta=(\alpha-1) / 2$. Отмеченный предельный переход дает также асимптотику $q_{n}\left(z^{2} / n^{2}\right) \sim j_{\beta}(z)$ равномерно по $z$ на компактных множествах действительной оси. Отсюда следует асимптотика ближайшего к началу нуля $\mu_{1, n}$ многочлена $Q_{n}(x): \mu_{1, n} \sim x_{1}^{2} / n^{2}$, где $x_{1}$ - ближайший к началу ноль функции Бесселя $J_{\beta}$, $\beta=(\alpha-1) / 2$, что доказывает первую часть теоремы. В случае весов Гегенбауэра рассуждения аналогичны, но требуют более точных оценок при выполнении предельного перехода.

Работа первого автора частично поддержана грантом научных школ 96-15-96185 и грантом РФФИ 99-01-01149. Работа третьего автора частично поддержана грантом РФФИ 99-01-01251 и грантом МОПО (Новосибирск).

\section{СПИСОК ЛИТЕРАТУРЫ}

[1] Ахиезер Н. И. Лекции по теории аппроксимаций. М.: Наука, 1972. [2] Milovanovic G.V., Mitrinovic D.S., Rassias Th. M. Topics in polynomials: extremal problems, inequalities, zeros: Word Scientific, 1994. [3] Draux A., Elhami Ch. // J. Comput. Appl. Math. 1999. V. 106. № 2. Р. 203-243. [4] Аптекарев А. И. // Матем. сб. 1992. Т. 183. № 5. С. 43-62.

Институт прикладной математики им. М.В. Келдыша РАН; $\quad$ Принято редколлегией INSA de Rouen, France;

Нижегородский государственный технический университет

E-mail: aptekaa@rfbr.ru, draux@insa-rouen.fr kalia@waise.nntu.sci-nnov.ru 